\title{
Uso de tecnologías de la información y comunicación (TIC) para mejorar la adherencia al tratamiento en pacientes con enfermedades crónicas
}

\author{
Use of Information and Communication Technology (ICT) to improve adherence to treatment in patients \\ with chronic diseases
}

\section{Sr. Editor}

Las enfermedades crónicas representan la principal causa de mortalidad en el mundo. Cerca del $63 \%$ del total de muertes durante el año 2008 se atribuyen especialmente a enfermedades cardiovasculares, diabetes, enfermedades respiratorias crónicas y cáncer (1). Un factor fundamental para reducir estas cifras, además de las medidas de promoción de la salud y prevención de la enfermedad, consiste en mejorar la adherencia al tratamiento, de modo que se logre disminuir el número de complicaciones y muertes asociadas a estas patologías (2).

Según la Organización Mundial de la Salud (OMS) el $50 \%$ de los pacientes que padecen enfermedades crónicas no presentan adherencia al tratamiento farmacológico (2). Para mejorar la adherencia al tratamiento de las enfermedades crónicas se han desarrollado principalmente intervenciones de tipo educativas, conductuales, combinadas, teniendo en cuenta el aspecto psicológico, la relación médico paciente, entre otras (3).

El desarrollo científico y tecnológico ha permitido desde la última década del siglo XX, la aplicación de las tecnologías de la información y comunicación (TIC) en el área de Salud, siendo herramientas novedosas en la búsqueda de mejorar la adherencia al tratamiento de las enfermedades. El uso de las TIC ha sido bien aceptado por médicos especialistas y también por los pacientes que padecen enfermedades crónicas, éstos refieren el interés que tienen en participar por ejemplo en el envío de mensajes a sus celulares para hacerles

Tabla 1. Estudios sobre el uso de las TIC para mejorar la adherencia al tratamiento en pacientes con enfermedades crónicas.

\begin{tabular}{lll}
\hline Autores & Grupo de estudio/Intervención & \multicolumn{1}{c}{ Resultados } \\
\hline Arora S , Peters AL, Agy & Pacientes con diabetes mellitus tipo 2/ & Se incrementó en 16,5\%, 30,5\% y 9\% el número \\
C, Menchine M (5). & Intervención: Envío de mensaje de texto. & $\begin{array}{l}\text { los sujetos que informaron comer frutas / verduras, } \\
\text { hacer ejercicios y realizar control de los pies, } \\
\end{array}$ \\
& & $\begin{array}{l}\text { Tiempo de intervención: 3 semanas. } \\
\text { Adherencia Morisky mejoró de 3,5 a 4,75. }\end{array}$
\end{tabular}

Warren J, Kennelly J, Pacientes con hipertensión arterial/ Warren D, Elley CR, Wai Intervención: Análisis de los registros KC, Manukia M, et. Al. médicos electrónicos (EMR). Tiempo de (6) intervención: 12 meses.

Vollmer WM, Feldstein A, Smith DH, Dubanoski JP, Waterbury A,

Schneider JL, et. Al. (7)
Pacientes con asma/Intervención: Uso del software de reconocimiento de voz interactiva (IVR).Tiempo de intervención: 18 meses.
De los 252 pacientes de intervención con tasa de posesión de medicamentos (MPR) $<80 \%$ inicialmente mejoró $(\mathrm{p}=0,0002)$ y la presión arterial sistólica disminuyó 3,5 mm Hg $(\mathrm{p}=0,07)$ en comparación con el grupo control.

Se obtuvo mayor adherencia significativa para los participantes del grupo de intervención en relación con los del grupo de atención habitual $(\Delta=0,02$, $95 \%$ intervalo de confianza 0,01 hasta 0,03$)$. 
recordar la toma de medicación o información sobre su enfermedad (4).

En la tabla 1 se presentan diversos estudios $(5,6,7)$ que muestran el uso de las TIC, a través del envío de mensajes de texto, análisis de los registros médicos electrónicos y el uso de software de reconocimiento de voz interactiva. Los estudios se realizaron en pacientes con diabetes mellitus tipo 2, hipertensión arterial y asma, respectivamente y los resultados reflejan incremento de la adherencia a la terapéutica atribuido a la intervención realizada.

A pesar de que la evidencia publicada sugiere que el uso de las TIC mejoraría la adherencia al tratamiento de las enfermedades crónicas, se debe tener en cuenta el seguimiento de los pacientes para poder observar la variación de la adherencia en etapas posteriores al uso de las TIC, además de determinar el tiempo necesario de intervención en un grupo de interés.

Un punto importante a tener en cuenta en el futuro es la brecha digital, que implica la separación que existe entre las personas, comunidades, estados, países que cuentan con la tecnología para su uso respecto de otros que pueden no tener los recursos tecnológicos o no poder emplearlos (8). Sin embargo, ante esta situación se vienen desarrollando acciones para lograr disminuirla (9).

El uso de las TIC podría ser útil como herramienta del sistema de salud en la búsqueda de una mayor participación del paciente en la mejora de su salud, mejorando la adherencia al tratamiento de las enfermedades crónicas cuyas prevalencias están en aumento en la actualidad, siendo importante el desarrollo de trabajos con un seguimiento amplio a los grupos intervenidos para evaluar la utilidad y solvencia a largo plazo de este tipo de intervención.

Sofía Prado-Cucho

Sociedad Científica de Estudiantes de Medicina de Ica (SOCEMI). Universidad Nacional San Luis Gonzaga de Ica. Perú.

Guido Bendezú-Quispe Sociedad Científica de Estudiantes de Medicina de Ica (SOCEMI) Universidad Nacional San Luis Gonzaga de Ica. Perú. Consejería Regional Centro, Sociedad Científica Médico Estudiantil Peruana (SOCIMEP).

\section{Correspondencia:}

Sofía Prado Cucho

Conjunto Habitacional Fonavi La Angostura. Primera Etapa R-8.

Subtanjalla. Ica.

Teléfonos: (56) 315254 - 956791514

Correo electrónico: sofialpradoc21@gmail.com

\section{REFERENCIAS BIBLIOGRÁFICAS}

1. Organización Mundial de la Salud. Informe sobre la situación mundial de las Enfermedades No Trans misibles 2010 - Resumen de Orientación. Ginebra: OMS; 2011.

2. Organización Mundial de la Salud. Adherencia a los tratamientos a largo plazo. Pruebas para la acción. Ginebra: OMS; 2004.

3. Kripalani S, Yao X, Haynes RB. Interventions to en hance medication adherence in chronic medical con ditions: a systematic review. Archives of internal medicine. 2007; 167 (6):540-50.

4. Curioso WH. Uso y percepciones hacia las tecnologías de información y comunicación en pacientes con diabetes, en un hospital público del Perú. Rev Perú Med Exp Salud Pública. 2009; 26(2): 161-167.

5. Arora S, Peters AL, Agy C, Menchine M. A mobile health intervention for inner city patients with poorly controlled diabetes: proof-of-concept of the TExTMED program. Diabetes Technol Ther. 2012; 14 (6):492-496.

6. Warren J, Kennelly J, Warren D, et al. Using the general practice EMR for improving blood pressure medication adherence. Stud Health Technol Inform. 2012; 178:228-234.

7. Vollmer WM, Feldstein A, Smith DH, et al. Use of health information technology to improve medication adherence. Am J Manag Care. 2011; 17(12 Spec No.):SP79-87.

8. Serrano A, Martinez E. La Brecha digital: mitos y realidades. Mexico DF: Editorial UABC; 2003. p. 1719.

9. Villatoro P, Silva A. Estrategias, programas y experiencias de superación de la brecha digital y universalización del acceso a las nuevas tecnologías de la información y la comunicación (TIC). Un panorama regional; Santiago de Chile: Comisión Económica para América Latina y el Caribe (CEPAL). Febrero de 2005.

Recibido: 27/01/13 